\title{
Realization of High-Quality Structural Color by Metal-Dielectric-Metal Structure
}

\author{
Woong Ki Jang', Yoo Su Kang', Young Ho Seo ${ }^{1}$ and Byeong Hee Kim¹ \\ ${ }^{1}$ Dept. of Convergence system Engineering / Kangwon National University \\ Chuncheon Gangwon-do 24341, Republic of Korea \\ wkddndrl@kangwon.ac.kr; kus2172@kangwon.ac.kr; mems@kangwon.ac.kr; kbh@kangwon.ac.kr
}

\section{Extended Abstract}

The present study produced a metal-ceramic and air-ceramic complex thin film that has a nanohole structure of various sizes using anodization and metal sputtering in combination, and created a high-quality structural color by converting the complex thin film with a target effective refractive index into a multi-layer thin film.

First, by applying the effective-medium theory, the nanohole structured thin film was assumed as a metal-ceramic or air-ceramic complex thin film with a new effective refractive index instead of the unique refractive index of the thin film. The effective refractive index was calculated using the Maxwell-Garnett equation [1] and the peak wavelength of the complex thin film's reflective structural color spectrum was calculated based on the thin-film interference theory. A metal-dielectricmetal (MDM) structure was created by vapor-depositing a metal on the surface of a nanohole-structured complex thin film to improve the quality of low-quality reflective structural color and the improvement was verified through experiments. The complex thin film of a nanohole array structure was produced by altering the factors affecting the refractive index, such as the nanohole's diameter and thickness as well as the distance between the nanoholes. The distance between the nanoholes was altered by changing the electrolyte and the magnitude of applied voltage used in the anodization process. The nanohole's diameter was modified through the widening process using alumina etching. The distance between the nanoholes could be altered within the range between $30 \mathrm{~nm}$ and $500 \mathrm{~nm}$ depending on the conditions of electrolyte and applied voltage, while the nanohole's diameter could be changed within the range between $10 \mathrm{~nm}$ and $400 \mathrm{~nm}$. The thickness of a nanohole array was altered according to the growth rate by controlling the total size of applied electric charge using the real time current monitoring system.

For the complex thin film simplified into a single medium, the peak wavelength of a reflective structural color spectrum was calculated based on the multi-layer interference theory. To compare the calculated value with the experimental value, this study measured the reflective structural color spectrum of the visible ray field using the halogen light source in the produced complex thin film. The two values differed by $1.9 \mathrm{~nm} \sim 18.7 \mathrm{~nm}$ and this represents an average difference of $1.3 \%$ compared to the difference of the maximum wavelength, confirming that the two values were considerably similar to each other.

The observation of MDM-structured reflective structural color by marking it on the color coordinate system showed that the quality of reflective structural color improved and confirmed that as the diameter and thickness of a nanohole in the complex thin film increase, the reflective structural color changes while rotating anticlockwise on the CIE color coordinate system.

\section{Acknowledgements}

This research was supported by Industry and Energy and Basic Science Research Program through the National Research Foundation of Korea (NRF) funded by the Ministry of Education (NRF-2017R1D1A1B03036123) and this research was funded and conducted under [the Competency Development Program for Industry Specialists] of the Korean Ministry of Trade, Industry and Energy (MOTIE), operated by Korea Institute for Advancement of Technology (KIAT).(P0002092) 


\section{References}

[1] J. M. Garnett, "VII. Colours in metal glasses, in metallic films, and in metallic solutions.-II," Phil. Trans. R. Soc. Lond. A, no. 205(387-401), pp. 237-288, 1906. 